\title{
Design of Group Precoding for MU-MIMO Systems with Exponential Spatial Correlation Channel
}

\author{
Van-Khoi Dinh ${ }^{1, *}$, Thanh-Binh $\mathrm{Chu}^{2}$, Minh-Tuan $\mathrm{Le}^{2}$ and Vu-Duc Ngo ${ }^{3}$ \\ ${ }^{1}$ Le Quy Don Technical University, ${ }^{2}$ MobiFone R\&D Center, MobiFone Corporation, ${ }^{3}$ Hanoi University of Science \\ and Technology
}

\section{Abstract}

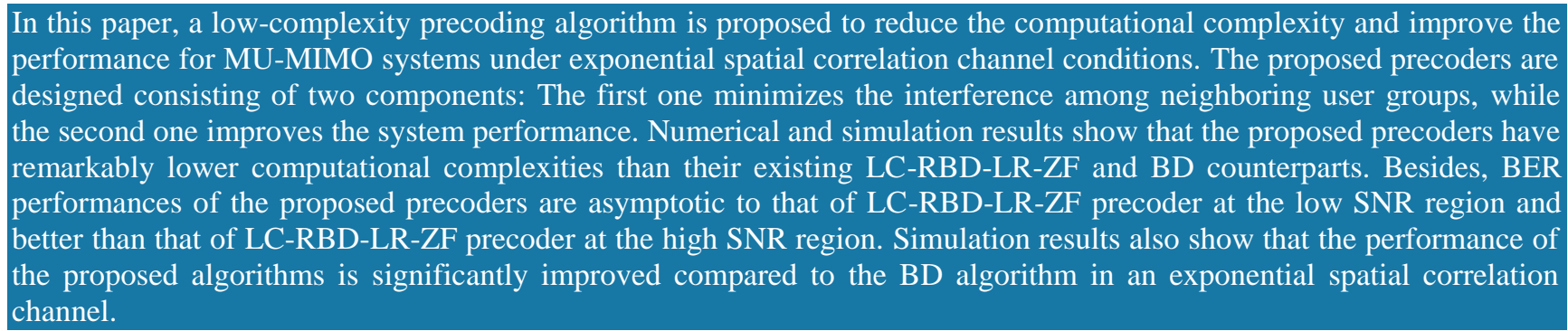

Keywords: MU-MIMO system, linear precoding algorithms, lattice reduction algorithms.

Received on 13 December 2020, accepted on 19 January 2021, published on 26 January 2021

Copyright (C) 2021 Van-Khoi Dinh et al., licensed to EAI. This is an open access article distributed under the terms of the Creative Commons Attribution licence (http://creativecommons.org/licenses/by/3.0/), which permits unlimited use, distribution and reproduction in any medium so long as the original work is properly cited.

doi: 10.4108/eai.26-1-2021.168228

\section{Introduction}

Multiple-Input Multiple-Output (MIMO) system has been widely studied in recent years and already applied in 4G mobile communication systems due to the fact that they can greatly increase the spectrum efficiency [1]. In order to utilize multiplexing gain, Multiuser MIMO (MUMIMO) system has been proposed. In the MU-MIMO, each base station (BS) is equipped with multi antennas to simultaneously serve multi users using the same frequency resource. MU-MIMO not only inherits all the advantages of MIMO systems but also overcomes its limitation [2].

Unlike single user MIMO (SU-MIMO) systems, the received signals at the user sides of MU-MIMO systems not only suffer from noise and inter-antenna interference but also affected by the interference among neighboring users. In order to solve this problem, precoding techniques are applied at the BS side. Linear precoding algorithms with low-complexity such as Zero Forcing

*Corresponding author. Email: Vankhoi.tcu@gmail.com
(ZF), Minimum Mean Square Error (MMSE) and Maximum Ratio Transmission (MRT) are suitable candidates [3], [4]. As shown in [2], when the number of antennas at the BS side is greater than the number of users, the simple linear precoders become nearly optimal. Obviously, nonlinear algorithms, such as Dirty Paper Coding (DPC) proposed in [5], can also be applied. However, the complexity of these algorithms becomes significantly large as the system dimensions grow due to the implementation of random nonlinear encoding and decoding [6], [7].

To improve the system performance, in [8], the authors combined Seysen's lattice reduction algorithm (SA) and linear precoding techniques for MU-MIMO systems. It is shown in [8] that the proposed algorithm gives better performance than the precoding algorithm with the Lenstra-Lenstra-Lovász (LLL) lattice reduction algorithm. In [9], Block Diagonalization (BD) precoding algorithm was proposed by combining $\mathrm{QR}$ decomposition and Pseudo-Inverse Block Diagonalization (PINV-BD) in [10]. In this proposal, LLL lattice reduction (LR) algorithm and Tomlinson-Halashima precoder (THP) are 
applied in each block to improve the quality of the system. In [11], the authors proposed the low-complexity lattice reduction-aided regularized Block Diagonalization using Zero Forcing precoding (e.g., LC-RBD-LR-ZF) and low-complexity lattice reduction-aided regularized Block Diagonalization using MMSE precoders (e.g., LC-RBDLR-MMSE) for MU-MIMO systems. In this proposal, the first precoding matrix is obtained by using $Q R$ decomposition of the channel matrix. The second one is designed based on ZF or MMSE algorithm in combination with LLL lattice reduction algorithm. The precoders in [9] and [11] were shown to significantly improve system performance. However, their computational complexity is still very high due to the adoptions of QR decomposition and LR algorithms. In [12], the precoding algorithm is proposed based on system expansion. Besides, the precoding algorithm based on the principal component analysis technique (PCA) is proposed for Massive MIMO systems [13]. However, for the proposals in [12] and [13], the authors have not given the symbol error probability analysis expression of the system.

In this paper, we propose two linear group precoding algorithms, called BD-LR-ZF and BD-LR-MMSE precoders, that have low complexity for MU-MIMO systems working in the exponential correlation channel model. In our proposal, the channel matrix from the BS to all users is divided into two groups, each group consists of a number of rows of the channel matrix. Based on this grouping approach, the proposed precoders are designed consisting of two components. The first precoding matrix minimizes the interference from neighboring user groups by using traditional $\mathrm{BD}$ algorithm; the second one improves the BER performance of the system by combining the conventional linear precoders and the element-base lattice reduction shortest longest basis (ELR-SLB) technique. Performance evaluation by analyzing the so-called orthogonal deficiency (od) component is provided so that one can roughly estimate and compare the performances among precoders. Numerical results are also provided to show that the proposed precoders have remarkably lower computational complexities than both the LC-RBD-LR-ZF in [11] and the BD in [4]. Simulation results show that the BER performance of the proposed algorithm is asymptotic to that of the LC-RBD-LR-ZF algorithm and better than the $\mathrm{BD}$ algorithm. Moreover, the spatial correlation adversely affects the system performance no matter which precoder is adopted.

The rest of this paper is organized as follows. In Section II, we present MU-MIMO system model. The proposed algorithms in combination with ELR-SLB technique are presented in Section III. In Section IV, we present the simulation results. Finally, conclusions are drawn in Section V.

Notation: The notations are defined as follows: Matrices and vectors are represented by symbols in bold; $(.)^{T}$ and $(.)^{H}$ denote the transpose and conjugate transpose, respectively. We denote $|\alpha|$ for the absolute value of scalar $\alpha$ and $\operatorname{det}(\mathbf{B})$ for the determinant of $\mathbf{B}$. $\operatorname{Tr}\{$.$\} is the trace of a square matrix, E\{$.$\} denotes the$ expectation operator. $\mathbf{A}^{H}, \mathbf{A}^{T}$ and $\mathbf{A}^{-1}$ are used to denote conjugate transpose, transpose, and inverse of $\mathbf{A}$, respectively. $\lfloor\alpha\rceil$ is to round the real and imaginary parts of the complex number to the nearest integer.

\section{The downlink channel model in MU- MIMO systems}

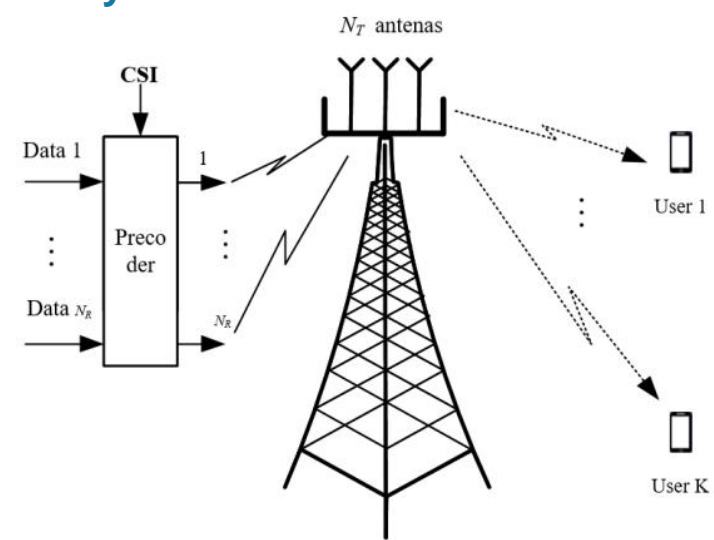

Fig. 1. The downlink channel model in MU-MIMO system

Let us consider a MU-MIMO system illustrated in Fig. 1, where the $\mathrm{BS}$ is equipped with $N_{T}$ antennas to simultaneously serve $K$ users. Each user has $N_{u}$ antennas. Thus, the total number of antennas of $K$ users is $N_{R}=K N_{u}$. In addition, the Channel State Information (CSI) is assumed to be perfectly known at the BS.

In real applications, both the BS side and user side do not have large spaces to arrange the antenna elements distant enough. Therefore, spatial correlations always exist among transmit and receive antennas, resulting in performance degradation. In order to take into account the effect of spatial correlation, the channel model is given by the following equation [14]:

$$
\mathbf{H}=\mathbf{R}_{R}^{1 / 2} \tilde{\mathbf{H}} \mathbf{R}_{T}^{1 / 2},
$$

where $\mathbf{H} \in \mathbb{C}^{N_{R} \times N_{T}}$ is the channel matrix with antenna correlation, $\mathbf{R}_{T}$ is the $N_{T} \times N_{T}$ transmit correlation matrix and $\mathbf{R}_{R}$ is the $N_{R} \times N_{R}$ receive correlation matrix. $\tilde{\mathbf{H}}$ is the uncorrelated channel matrix, whose entries, $\tilde{\mathbf{h}}_{i j}$, are complex Gaussian random variables with zero mean and unit variance. In this paper, we investigate MUMIMO systems in exponentially correlated channel model [15]. In this model, the components of $\mathbf{R}_{T}$ and $\mathbf{R}_{R}$ are determined as follows:

$$
r_{m n}=\left\{\begin{array}{l}
r^{n-m}, m \leq n \\
r_{n m}^{*}, n>m
\end{array},|r| \leq 1,\right.
$$

herein $r \geq 0$ is the correlation coefficient between any two neighboring antenna elements. 
Let $\mathbf{y}=\left[\begin{array}{llll}\mathbf{y}_{1}^{T} & \mathbf{y}_{1}^{T} & \cdots & \mathbf{y}_{K}^{T}\end{array}\right]^{T} \in \mathbb{C}^{N_{R} \times 1}$ be the overall received signal vector for all users. Then, the relationship between the transmitted signal vector, $\mathbf{x}=\left[\mathbf{x}_{1}^{T} \mathbf{x}_{2}^{T} \ldots \mathbf{x}_{K}^{T}\right]^{T}$ $\in \mathbb{C}^{N_{R} \times 1}$ and the received signal vector $\mathbf{y}$ is given by:

$$
\mathbf{y}=\mathbf{H W x}+\mathbf{n},
$$

where $\mathbf{H}$ is channel matrix from BS to all $K$ users, defined in (1). $\mathbf{W} \in \mathbb{C}^{N_{T} \times N_{R}}$ is the precoding matrix to be designed for all users. $\mathbf{n} \in \mathbb{C}^{N_{R} \times 1}$ is noise vector at the $K$ users, whose entries are assumed to be identical independent distributed (i.i.d) random variables with zero mean and variance $\sigma_{n}^{2}$.

\section{Proposed algorithm}

\subsection{Proposed BD-LR-ZF and BD-LR- MMSE algorithms}

In this section, based on the BD method in [4], we present a linear group precoding method in combination with the low-complexity ELR-SLB lattice reduction technique in [16] for the MU-MIMO systems.

The overall precoding matrix for all users is defined as follows:

$$
\mathbf{W}=\beta \mathbf{W}_{B D} \mathbf{W}_{L P},
$$

herein $\mathbf{W}_{B D} \in \mathbb{C}^{N_{T} \times N_{R}}$ is designed to minimize the interferences from other user groups and $\mathbf{W}_{L P} \in \mathbb{C}^{N_{R} \times N_{R}}$ is designed to enhance the system performance. $\beta$ is the normalized power factor.

In the first step, the correlation channel matrix $\mathbf{H}$ is divided into two groups (i.e., sub-matrices) $\mathbf{H}_{1} \in \mathbb{C}^{a \times N_{T}}$ and $\mathbf{H}_{2} \in \mathbb{C}^{\alpha \times N_{T}}, \alpha=N_{R} / 2$. In the case with an odd value for $N_{R}, \alpha$ is rounded up or down to the nearest integer. The first group $\mathbf{H}_{1}$, consists of the first row to the $\alpha$ th row of the channel matrix $\mathbf{H}$; the second group $\mathbf{H}_{2}$, is from the $(\alpha+1)$ th row to the $N_{R}$ th row. Specifically, the correlation channel matrix from BS to all users can be represented as follows:

$$
\mathbf{H}=\left[\begin{array}{l}
\mathbf{H}_{1} \\
\mathbf{H}_{2}
\end{array}\right] .
$$

The precoding matrix $\mathbf{W}_{B D}$ is determined in the next step. Applying Singular Value Decomposition (SVD) to $\mathbf{H}_{2}$, we get:

$$
\mathbf{H}_{2}=\mathbf{S}_{1} \mathbf{V}_{1} \mathbf{D}_{1}
$$

where $\mathbf{V}_{1} \in \mathbb{C}^{\alpha \times N_{T}}$ is a diagonal matrix. $\mathbf{S}_{1} \in \mathbb{C}^{\alpha \times a}$ and $\mathbf{D}_{l} \in \mathbb{C}^{N_{T} \times N_{T}}$ are unitary matrices with orthogonal columns. The precoding matrix $\mathbf{W}_{B D}^{1} \in \mathbb{C}^{N_{T} \times \alpha}$ for the first group is constructed as:

$$
\mathbf{W}_{B D}^{1}=\mathbf{D}_{1}\left(:, \alpha+1: N_{T}\right)
$$

Applying the same steps to $\mathbf{H}_{1}$, we are able to get the next precoding matrix $\mathbf{W}_{B D}^{2}$. The precoding matrix $\mathbf{W}_{B D}$ is designed to have the following form:

$$
\mathbf{W}_{B D}=\left[\begin{array}{ll}
\mathbf{W}_{B D}^{1} & \mathbf{W}_{B D}^{2}
\end{array}\right] \text {. }
$$

After getting the first weight matrix $\mathbf{W}_{B D}$, we define the effective channel matrix for the first group as follows:

$$
\overline{\mathbf{H}}_{1}=\mathbf{H}_{1} \mathbf{W}_{B D}^{1} .
$$

The channel matrix $\overline{\mathbf{H}}_{1}$ is then transposed and converted into the matrix $\overline{\mathbf{H}}_{1}^{L R} \in \mathbb{C}^{\alpha \times \alpha}$ in the LR domain by using ELR-SLB algorithm in [16] to give:

$$
\overline{\mathbf{H}}_{1}^{L R}=\mathbf{U}_{1}^{T} \overline{\mathbf{H}}_{1},
$$

herein $\mathbf{U}_{1}^{T}$ is a unimodular matrix with integer elements $\left(\operatorname{det}\left|\mathbf{U}_{1}^{T}\right|=1\right)$.

The weight matrix $\mathbf{W}_{Z F}^{1}$ or $\mathbf{W}_{M M S E}^{1}$ for the first group is created by applying ZF or MMSE algorithm to $\overline{\mathbf{H}}_{1}^{L R}$ as follows:

$$
\begin{gathered}
\mathbf{W}_{Z F}^{1}=\left(\overline{\mathbf{H}}_{1}^{L R}\right)^{H}\left[\left(\overline{\mathbf{H}}_{1}^{L R}\right)\left(\overline{\mathbf{H}}_{1}^{L R}\right)^{H}\right]^{-1} \\
\mathbf{W}_{M M S E}^{1}=\left(\overline{\mathbf{H}}_{1}^{L R}\right)^{H}\left[\left(\overline{\mathbf{H}}_{1}^{L R}\right)\left(\overline{\mathbf{H}}_{1}^{L R}\right)^{H}+\sigma^{2} \mathbf{U}_{1} \mathbf{U}_{1}^{H}\right]^{-1}
\end{gathered}
$$

where $\sigma^{2}=\sigma_{n}^{2} / E_{s}, E_{s}$ is the energy of each transmit symbol. Following the same steps, we can create the precoding matrices $\mathbf{W}_{Z F}^{2}$ and $\mathbf{W}_{M M S E}^{2}$. Finally, the precoding matrix $\mathbf{W}_{L P}$ and the unimodular matrix $\mathbf{U}_{G P}$ for all groups can be obtained as follows:

$$
\mathbf{W}_{L P}=\left[\begin{array}{lr}
\mathbf{W}_{Z F}^{1} & 0 \\
0 & \mathbf{W}_{Z F}^{2}
\end{array}\right],
$$

or

$$
\mathbf{W}_{L P}=\left[\begin{array}{ll}
\mathbf{W}_{M M S E}^{1} & 0 \\
0 & \mathbf{W}_{M M S E}^{2}
\end{array}\right] .
$$




$$
\mathbf{U}_{G P}=\left[\begin{array}{cc}
\mathbf{U}_{1}^{T} & 0 \\
0 & \mathbf{U}_{2}^{T}
\end{array}\right]
$$

In order to make sure that the transmit power is unchanged after having precoded the transmit signals, the normalized power factor $\beta$ is computed as follows:

$$
\beta=\sqrt{\frac{N_{R}}{\operatorname{Tr}\left[\left(\mathbf{W}_{B D} \mathbf{W}_{L P}\right)\left(\mathbf{W}_{B D} \mathbf{W}_{L P}\right)^{H}\right]}} .
$$

The proposed BD-LR-ZF and BD-LR-MMSE algorithms are summarized in Algorithm 1.

Algorithm 1: The BD-LR-ZF and BD-LR-MMSE precoding algorithms

1. Input $N_{T}, N_{R}, \mathbf{H}$

2. Divide the matrix $\mathbf{H}$ into two groups as in (5).

3. Apply SVD decomposition to $\mathbf{H}_{2}$.

4. Generate the matrix $\mathbf{W}_{B D}^{1}=\mathbf{D}_{1}\left(:, \alpha+1: N_{T}\right)$.

5. Repeat Step 3 and Step 4 for the second user group.

6. Generate the matrix $\mathbf{W}_{B D}=\left[\mathbf{W}_{B D}^{1} \mathbf{W}_{B D}^{2}\right]$ as in (8).

7. Generate the matrix $\overline{\mathbf{H}}_{1}=\mathbf{H}_{1} \mathbf{W}_{B D}^{1}$.

8. Convert $\overline{\mathbf{H}}_{1}^{T}$ into $\overline{\mathbf{H}}_{1}^{L R}$ by the ELR-SLB algorithm in [16].

9. Create the matrix $\mathbf{W}_{Z F}^{1}$ or $\mathbf{W}_{M M S E}^{1}$ as in (11) and (12).

10. Repeat Step 7 to Step 9 for the second user group.

11. Generate the matrix $\mathbf{W}_{L P}$ as in (13) and (14).

12. Output: $\beta=\sqrt{\frac{N_{R}}{\operatorname{Tr}\left[\left(\mathbf{W}_{B D} \mathbf{W}_{L P}\right)\left(\mathbf{W}_{B D} \mathbf{W}_{L P}\right)^{H}\right]}}$.

$\mathbf{W}=\beta \mathbf{W}_{B D} \mathbf{W}_{L P}$

At the user side, the received signal vector is expressed as:

$$
\mathbf{y}=(\mathbf{H W x}+\mathbf{n}) / \beta .
$$

The received signal $\mathbf{y}$ is then quantized to the nearest constellation symbols to give the recovered signals for all users.

\subsection{Performance Analysis}

From (17), the estimated signal vector of all users is given by:

$$
\tilde{\mathbf{x}}=\mathbf{U}_{G P} \frac{1}{\alpha}\left(\left\lfloor\alpha \mathbf{y}+\beta_{z}\left(\mathbf{U}_{G P}\right)^{-1} \mathbf{1}_{L}\right\rceil-\beta_{z}\left(\mathbf{U}_{G P}\right)^{-1} \mathbf{1}_{L}\right),
$$

where $\quad \alpha=1 / 2, \beta_{z}=\frac{m-1}{2}(1+j), \mathbf{1}_{L} \in R^{N_{R} \times 1} \quad$ is $\quad$ a column vector with $N_{R}$ ones, $m$ is the number of bits in a transmitted symbol.

Substituting $\mathbf{y}$ in (17) to (18), we can obtain:

$$
\tilde{\mathbf{x}}=\mathbf{x}+2 \mathbf{U}_{G P} Q_{z}\left[\frac{1}{2} \frac{\mathbf{n}}{\beta}\right]
$$

herein $Q_{z}[a]$ denotes the operation that rounds $a$ to the nearest integer. Apparently, if $Q_{z}\left[\frac{1}{2} \frac{\mathbf{n}}{\beta}\right]=\mathbf{0}, \mathbf{x}$ will be decoded correctly. Therefore, the symbol error probability (SEP) for a given $\mathbf{H}$ is upper-bounded by:

$$
\begin{aligned}
P_{e \mid \mathbf{H}} & \leq 1-P\left(Q_{z}\left[\frac{1}{2} \frac{\mathbf{n}}{\beta}\right]=\mathbf{0} \mid \mathbf{H}\right) \\
& \leq 1-P\left(Q_{z}\left[\sqrt{\operatorname{Tr}\left[\mathbf{W} \mathbf{W}^{H}\right]}\right]=\mathbf{n} \mid \mathbf{H}\right),
\end{aligned}
$$

herein $\quad \mathbf{W}=\mathbf{W}_{B D} \mathbf{W}_{L P}$. Let us denote $\mathbf{W}=\left[\mathbf{w}_{1}, \mathbf{w}_{2}, \ldots, \mathbf{w}_{N_{T}}\right]^{T}$, where $\mathbf{w}_{i}^{T} i \in 1, N_{T} \quad$ is the $i$ th row of $\mathbf{W}$. The upper bound is rewritten as follows:

$$
\begin{aligned}
P_{e \mid \mathbf{H}} & \leq P\left(\max _{1 \leq i \leq N_{T}} \sqrt{\sum_{i=1}^{N_{T}}\left\|\mathbf{w}_{i}^{T}\right\|^{2}} \mathbf{n} \geq \frac{1}{2} \mid \mathbf{H}\right) \\
& \leq P\left(\max _{1 \leq i \leq N_{T}}\left|\mathbf{w}_{i}^{T} \mathbf{n}\right| \geq \frac{1}{2} \mid \mathbf{H}\right) .
\end{aligned}
$$

From [17], we have:

$$
\max _{1 \leq i \leq N_{T}}\left\|\mathbf{w}_{i}^{T}\right\| \leq \frac{1}{\sqrt{1-\operatorname{od}(\mathbf{W})} \cdot \min _{1 \leq k \leq N_{R}}\left\|\tilde{\mathbf{w}}_{k}\right\|},
$$

where $\tilde{\mathbf{w}}_{k}$ is the $k$ th column of $\mathbf{W} \cdot \operatorname{od}(\mathbf{W})$ is an orthogonality deficiency of the matrix $\mathbf{W}$ defined as [16], [18]:

$$
\operatorname{od}(\mathbf{W})=1-\frac{\operatorname{det}\left(\mathbf{W}^{H} \mathbf{W}\right)}{\prod_{k=1}^{N_{R}}\left\|\tilde{\mathbf{W}}_{k}\right\|^{2}}
$$

Note that, $0 \leq \operatorname{od}(\mathbf{W}) \leq 1$. If $\mathbf{W}$ is singular, $\operatorname{od}(\mathbf{W})=1$, and if $\mathbf{W}$ is orthogonal, $\operatorname{od}(\mathbf{W})=0$.

Using to the property of a norm, we can write: 


$$
\begin{aligned}
\max _{1 \leq i \leq N_{T}}\left|\mathbf{w}_{i}^{T} \mathbf{n}\right| & \leq \max _{1 \leq i \leq N_{T}}\left\|\mathbf{w}_{i}^{T}\right\| \cdot\|\mathbf{n}\| \\
& \leq \frac{\|\mathbf{n}\|}{\sqrt{1-\operatorname{od}(\mathbf{W})} \cdot \min _{1 \leq k \leq N_{R}}\left\|\tilde{\mathbf{w}}_{k}\right\|} .
\end{aligned}
$$

From (24) and (21), it follows that:

$$
\begin{aligned}
P_{e \mid \mathbf{H}} & \leq P\left(\frac{2\|\mathbf{n}\|}{\sqrt{1-o d(\mathbf{W})} \cdot \min _{1 \leq k \leq N_{R}}\left\|\tilde{\mathbf{w}}_{k}\right\|} \geq 1 \mid \mathbf{H}\right) \\
& \leq P \quad 2\|\mathbf{n}\| \geq \sqrt{1-o d(\mathbf{W})} \cdot \min _{1 \leq k \leq N_{R}}\left\|\tilde{\mathbf{w}}_{k}\right\| \mid \mathbf{H} .
\end{aligned}
$$

By averaging (25) over all realization of $\mathbf{H}$, the average symbol error probability is obtained as follows:

$$
\begin{aligned}
P_{e} & =E_{\mathbf{H}}\left[P_{e \mid \mathbf{H}}\right] \\
& \leq E_{\mathbf{H}}\left[P \quad 4\|\mathbf{n}\|^{2} \geq \sqrt{1-\operatorname{od}(\mathbf{W})}^{2} \times\right. \\
& \left.\min _{1 \leq k \leq N_{R}}\left\|\tilde{\mathbf{w}}_{k}\right\|^{2} \mid \mathbf{H}\right] \\
& =E_{\mathbf{n}}\left[P\left\{\min _{1 \leq k \leq N_{R}}\left\|\tilde{\mathbf{w}}_{k}\right\|^{2} \leq \frac{4\|\mathbf{n}\|^{2}}{1-\operatorname{od}(\mathbf{W})} \mid \mathbf{n}\right\} .\right.
\end{aligned}
$$

As show in [16], we have $P \min _{1 \leq k \leq N_{R}}\left\|\tilde{\mathbf{w}}_{k}\right\|^{2} \leq \mu \leq \varphi_{N_{R}, N_{T}} \mu^{N_{R}}$, where $\varphi_{N_{R}, N_{T}}$ is a finite constant depending on $N_{R}$ and $N_{T}$. Therefore, the average error probability in (26) is bounded as:

$$
\begin{aligned}
P_{e} & \leq E_{\mathbf{n}}\left[P\left\{\min _{1 \leq k \leq N_{R}}\left\|\tilde{\mathbf{w}}_{k}\right\|^{2} \leq \frac{4\|\mathbf{n}\|^{2}}{1-o d(\mathbf{W})} \mid \mathbf{n}\right\}\right] \\
& \leq E_{\mathbf{n}}\left[\varphi_{N_{R}, N_{T}}\left(\frac{4}{1-o d(\mathbf{W})}\right)^{N_{R}}\|\mathbf{n}\|^{2 N_{R}}\right] \\
& =\varphi_{N_{R}, N_{T}}\left(\frac{4}{1-E[\operatorname{od}(\mathbf{W})]}\right)^{N_{R}} \times \frac{2 N_{R}-1 !}{N_{R}-1 !}\left(\frac{1}{\sigma_{n}^{2}}\right)^{-N_{R}},
\end{aligned}
$$

where the last equality comes from the $N_{R}$ th moment of Chisquare random variable $\|\mathbf{n}\|^{2}$ [19].

From (27), we can see that $E[\operatorname{od}(\mathbf{W})]$ plays a critical role in the SEP of MU-MIMO system. Specifically, the SEP of the system increases as $E[\operatorname{od}(\mathbf{W})]$ increases and vice versa.

In Fig. 2, different curves of $E[\operatorname{od}(\mathbf{W})]$ are shown for the proposed algorithms and BD, LC-RBD-LR-ZF algorithms working in correlated channels (i.e., $\left.\mathbf{H}=\mathbf{R}_{R}^{1 / 2} \tilde{\mathbf{H}} \mathbf{R}_{T}^{1 / 2}\right)$ at $\mathrm{SNR}=27 \mathrm{~dB}$. Here, $N_{R}=N_{T}=6$ and the channel correlation coefficient is changed within the range $r=0 \rightarrow 0.9$. The simulation results in Fig. 2 show that $E[\operatorname{od}(\mathbf{W})]$ increases as the correlation coefficient increases.

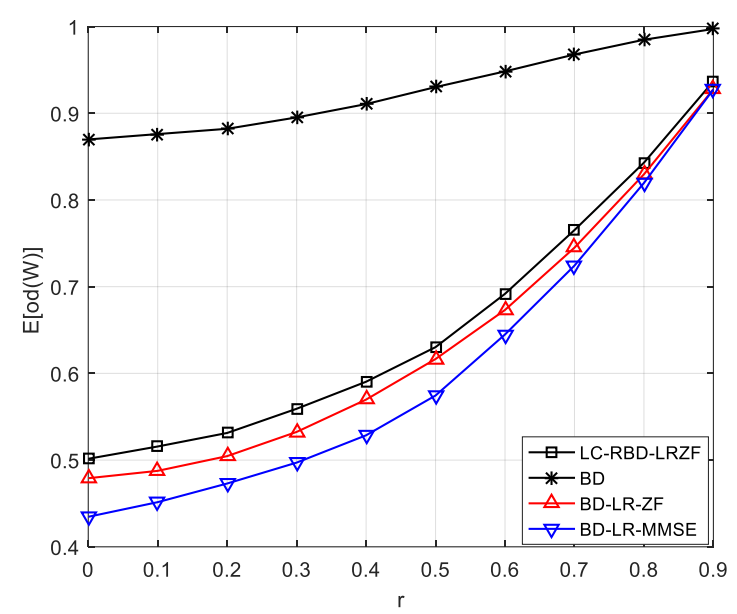

Fig. 2. $E[\operatorname{od}(\mathbf{W})]$ for BD-LR-ZF, BD, BD-LR-ZF and LC-RBD-LR-MMSE algorithms with $N_{R}=N_{T}=6$ and $r=0 \rightarrow 0.9$.

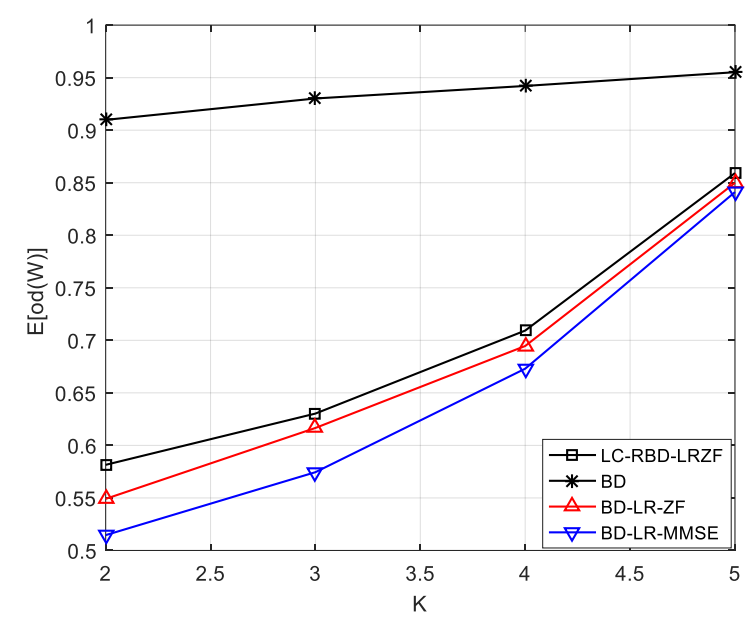

Fig. 3. $E[\operatorname{od}(\mathbf{W})]$ for BD-LR-ZF, BD, BD-LR-ZF and LC-RBD-LR-MMSE algorithms with $r=0.5, K=2,3,4,5$

$$
\text { and } N_{R}=N_{T} \text {. }
$$

Fig. 3 illustrates the curves of as functions of $K$ for all algorithms at $\mathrm{SNR}=27 \mathrm{~dB}$, và $K=2,3,4,5$. It can be observed from Fig. 3 that increases in an exponential manner with respect to $K$, i.e., with respect to the system size. More importantly, we can see from both figures that both BD-LR-MMSE and LC-RBD-LR-ZF precoders always have smaller than the remaining precoders. This means that, BD-LR-MMSE and LC-RBD-LR-ZF 
precoders will outperform their BD-LR-ZF and BD counterpart regarding the SEPs, as confirmed by the simulation results in the below section.

\subsection{Computational Complexity Analysis}

In this sub section, we evaluate the computational complexity of the proposed precoders and compare them with those of LC-RBD-LR-ZF algorithm in [11] and of BD algorithm in [4]. The complexities are evaluated by counting the necessary floating point operations (flops). We assume that each real operation (such as an addition, a multiplication or a division) is counted as a flop. Hence, a complex multiplication and a division require 6 flops and 11 flops, respectively. According to [20], SVD operation of an $m \times n$ complex matrix with $m<n$ requires $4 n^{2} m+8 n m^{2}+9 m^{3}$ flops.

Based on the above assumptions, the computational complexities of the proposed BD-LR-ZF and BD-LRMMSE precoders are given by:

$$
F=F_{1}+F_{2}+F_{3}+F_{4}+F_{5} \text { (flops) }
$$

where $F_{1}$ is the number of flops required for SVD operation of the $\tilde{\mathbf{H}}_{l}$ matrix; $F_{2}$ is the number of flops of the multiplication two matrices $\mathbf{H}_{l}$ and $\mathbf{W}_{B D}^{l}(l=1,2)$; $F_{3}$ is the number of flops to create $\overline{\mathbf{H}}_{l}^{L R}$ by the ELR-SLB algorithm in [14]; $F_{4}$ is the number of flops to create the matrix $\mathbf{W}_{Z F}^{l}$ or $\mathbf{W}_{M M S E}^{l}$ in (11) and (12), respectively; and $F_{5}$ is the number of flops for the multiplication two matrices $\mathbf{W}_{B D}$ and $\mathbf{W}_{L P}$.

The number of flops for SVD operations is given by:

$$
F_{1}=2\left(4 N_{T}^{2} \alpha+8 N_{T} \alpha^{2}+9 \alpha^{3}\right)(\text { flops })
$$

$F_{2}$ is calculated to be:

$$
F_{2}=2\left(8 N_{T} \alpha^{2}-2 \alpha^{2}\right)(\text { flops })
$$

Since ELR-SLB algorithm is adopted, $F_{3}$ is given by:

$$
F_{3}=2\left(24 \alpha^{3}-4 \alpha^{2}+F_{S L B}\right)(\text { flops })
$$

herein $F_{S L B}$ is the number of flops for the update operation of ELR-SLB algorithm [14], which can only be obtained by using the computer simulation. Note that each update operation in ELR-SLB algorithm requires $(16 \alpha+8)$ flops. The computations of $\lambda_{i, k}$ and $\Delta_{i, k}$ of
ELR-SLB algorithm in [16] need 4 flops and 10 flops, respectively. Therefore, $F_{S L B}$ is calculated as follows:

$$
\begin{aligned}
F_{S L B}= & \text { CUpdate } \times(16 \alpha+8) \\
& + \text { CLamda } \times 4+\text { CDelta } \times 10(\text { flops }),
\end{aligned}
$$

where CLamda is the number of updates $\lambda_{i, k}$, CDelta is the number of updates $\Delta_{i, k}$, CUpdate is the number of updates $t_{k}^{\prime}$ and $\tilde{c}^{k}$ from Steps 7 to Step 9 of ELR-SLB algorithm in [14].

The computational complexity of $\mathrm{ZF}$ algorithm is the number of flops to calculate: $\quad\left(\overline{\mathbf{H}}_{1}^{L R}\right)\left(\overline{\mathbf{H}}_{1}^{L R}\right)^{H}$, $\left[\left(\overline{\mathbf{H}}_{1}^{L R}\right)\left(\overline{\mathbf{H}}_{1}^{L R}\right)^{H}\right]^{-1}$ and $\left(\overline{\mathbf{H}}_{1}^{L R}\right)^{H}\left[\left(\overline{\mathbf{H}}_{1}^{L R}\right)\left(\overline{\mathbf{H}}_{1}^{L R}\right)^{H}\right]^{-1}$ in (11). Therefore, $F_{4}$ is calculated to be:

$$
F_{4}=2\left(24 \alpha^{3}-4 \alpha^{2}\right)(\text { flops })
$$

The computational complexity of the MMSE algorithm is the number of flops to calculate: $\sigma^{2} \mathbf{U}_{1} \mathbf{U}_{1}^{H}$, $\left(\overline{\mathbf{H}}_{1}^{L R}\right)\left(\overline{\mathbf{H}}_{1}^{L R}\right)^{H}+\sigma^{2} \mathbf{U}_{1} \mathbf{U}_{1}^{H}, \quad\left[\left(\overline{\mathbf{H}}_{1}^{L R}\right)\left(\overline{\mathbf{H}}_{1}^{L R}\right)^{H}+\sigma^{2} \mathbf{U}_{1} \mathbf{U}_{1}^{H}\right]^{-1}$ and $\quad\left(\overline{\mathbf{H}}_{1}^{L R}\right)^{H}\left[\left(\overline{\mathbf{H}}_{1}^{L R}\right)\left(\overline{\mathbf{H}}_{1}^{L R}\right)^{H}+\sigma^{2} \mathbf{U}_{1} \mathbf{U}_{1}^{H}\right]^{-1} \quad$ in $\quad$ [12]. Therefore, in this case, $F_{4}$ can be obtained as follows:

$$
F_{4}=2\left(24 \alpha^{3}-3 \alpha^{2}+\alpha+1\right)(\text { flops })
$$

$F_{5}$ is given by:

$$
F_{5}=8 N_{T} N_{R}^{2}-2 N_{R}^{2} \text { (flops) }
$$

From the above analysis results, the total number of flops for the proposed BD-LR-ZF and BD-LR-MMSE precoders are given in (36) and (37), respectively.

$$
\begin{aligned}
F_{B D-L R-Z F} & =F_{1}+F_{2}+F_{3}+F_{4}+F_{5} \\
& =2\left(4 N_{T}^{2} \alpha+8 N_{T} \alpha^{2}+9 \alpha^{3}\right)+2\left(8 N_{T} \alpha^{2}-2 \alpha^{2}\right) \\
& +2\left[24 \alpha^{3}-4 \alpha^{2}+\text { CUpdate } \times(16 \alpha+8)\right. \\
& + \text { CLamda } \times 4+\text { CDelta } \times 10]+2\left(24 \alpha^{3}-4 \alpha^{2}\right) \\
& +8 N_{T} N_{R}^{2}-2 N_{R}^{2}(\text { flops })
\end{aligned}
$$

$$
\begin{aligned}
F_{B D-L R-M M S E} & =F_{1}+F_{2}+F_{3}+F_{4}+F_{5} \\
& =2\left(4 N_{T}^{2} \alpha+8 N_{T} \alpha^{2}+9 \alpha^{3}\right)+2\left(8 N_{T} \alpha^{2}-2 \alpha^{2}\right) \\
& +2\left[24 \alpha^{3}-4 \alpha^{2}+\text { CUpdate } \times(16 \alpha+8)\right. \\
& + \text { CLamda } \times 4+\text { CDelta } \times 10]+2\left(24 \alpha^{3}-3 \alpha^{2}+\alpha+1\right) \\
& \left.8 N_{T} N_{R}^{2}-2 N_{R}^{2} \text { (flops }\right)
\end{aligned}
$$

The complexities all of the precoders under consideration are summarized in Table I. 


\begin{tabular}{|c|c|c|}
\hline $\begin{array}{c}\text { Precoding } \\
\text { algorithms }\end{array}$ & Complexity (flops $)$ & $\begin{array}{c}\text { Complexity } \\
\text { level }\end{array}$ \\
\hline $\begin{array}{c}\text { LC-RBD-LR- } \\
\text { ZF }\end{array}$ & $\begin{array}{c}K\left[6\left(N_{R}-N_{u}\right)\left(N_{R}+N_{T}-N_{u}\right)^{2}+4\left(N_{R}-N_{u}\right)\left(N_{R}+N_{T}-N_{u}\right)-\left(N_{R}+N_{T}-N_{u}\right)^{2}\right. \\
\left.-\left(N_{R}+N_{T}-N_{u}\right)\right]+K\left(8 N_{T}^{2} N_{u}-2 N_{T} N_{u}\right)+K\left(16 N_{u}^{2} N_{T}-2 N_{u} N_{T}+8 N_{u}^{3}-2 N_{u}^{2}+F_{L L L}\right) \\
+K\left(8 N_{u}^{3}+16 N_{u}^{2} N_{T}-2 N_{u}^{2}-2 N_{u} N_{T}\right)+8 K N_{T}^{2} N_{R}-2 N_{T} N_{R}\end{array}$ & $O\left(K N_{T}^{2} N_{R}\right)$ \\
\hline BD & $K\left[4 N_{T}^{2}\left(N_{R}-N_{u}\right)+8 N_{T}\left(N_{R}-N_{u}\right)^{2}+9\left(N_{R}-N_{u}\right)^{3}\right]$ & $O\left(N_{T}^{2} N_{R}\right)$ \\
\hline BD-LR-ZF & $2\left(4 N_{T}^{2} \alpha+8 N_{T} \alpha^{2}+9 \alpha^{3}\right)+2\left(8 N_{T} \alpha^{2}-2 \alpha^{2}\right)+2\left[24 \alpha^{3}-4 \alpha^{2}+C U p d a t e \times(16 \alpha+8)\right.$ & $O\left(N_{T} N_{R}^{2}\right)$ \\
\hline $\begin{array}{c}\text { BD-LR- } \\
\text { MMSE }\end{array}$ & $2\left(4 N_{T}^{2} \alpha+8 N_{T} \alpha^{2}+9 \alpha^{3}\right)+2\left(8 N_{T} \alpha^{2}-2 \alpha^{2}\right)+2\left[24 \alpha^{3}-4 \alpha^{2}+C U_{p d a t e} \times(16 \alpha+8)\right.$ & $O\left(N_{T} N_{R}^{2}\right)$ \\
\hline
\end{tabular}

\section{Simulation Results}

In this Section, we compare both the computational complexities and the BER performances of the proposed algorithms with those of LC-RBD-LR-ZF algorithm in [11] and BD algorithm in [4]. In all simulation results, the channel from BS to all users are assumed to be quasistatic Rayleigh fading channel.

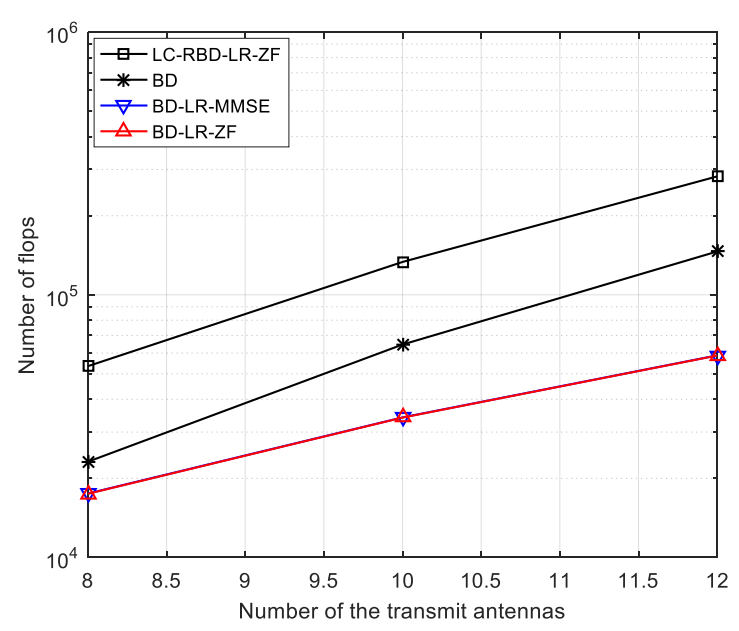

Fig. 4. Complexity comparison of all precoding algorithms

Fig. 4 demonstrates the computational complexities of LC-RBD-LR-ZF, BD, and the proposed precoders. In this scenario, $N_{T}$ is varied from 8 to 12 transmit antennas. It can be seen from the figure that the complexities of the proposed precoders are significantly lower than those of the LC-RBD-LR-ZF and the BD. For example, at $N_{R}=N_{T}=8$ antennas, the complexity of the proposed
BD-LR-MMSE is approximately equal to $32.6 \%$ and $75.5 \%$ of LC-RBD-LR-ZF and BD precoders' complexities, respectively.

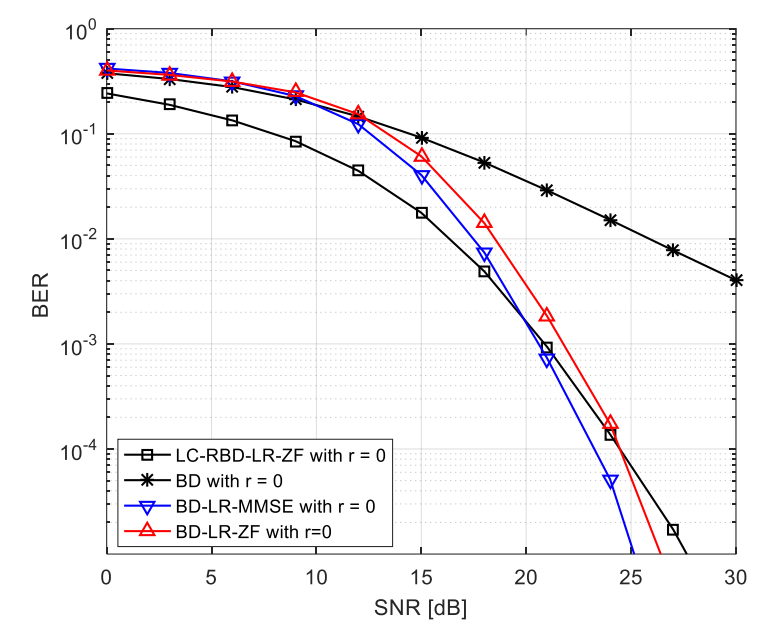

Fig. 5. The system performance with $N_{T}=8, N_{u}=2, K$ $=4$ in the case of uncorrelated channel

BER performances of all the precoding algorithms are illustrated in Fig. 5 to Fig. 7. In Fig. 5, the system is assumed to work in an uncorrelated MU-MIMO channel with the following parameters: $N_{T}=8, N_{u}=2, K=4$, and 4-QAM modulation. In Fig. 6, we simulate the system performance under the existence of exponential correlation at both the BS side and the user side (i.e., $\left.\mathbf{H}=\mathbf{R}_{R}^{1 / 2} \tilde{\mathbf{H}} \mathbf{R}_{T}^{1 / 2}\right)$. The correlation coefficients are assumed to be $r=0.5$ and $r=0.7$. Other parameters are the same as those used to generate Fig. 5. It can be seen from both Fig. 5 and Fig. 6 that in the low and medium SNR 
regions, the proposed BD-LR-ZF and BD-LR-MMSE precoders underperform their LC-RBD-LR counterpart. However, at sufficiently high SNRs, they provide better system performance than LC-RBD-LR-ZF precoder. More importantly, in all scenarios, the proposed precoders outperform the BD one in the entire SNR region.

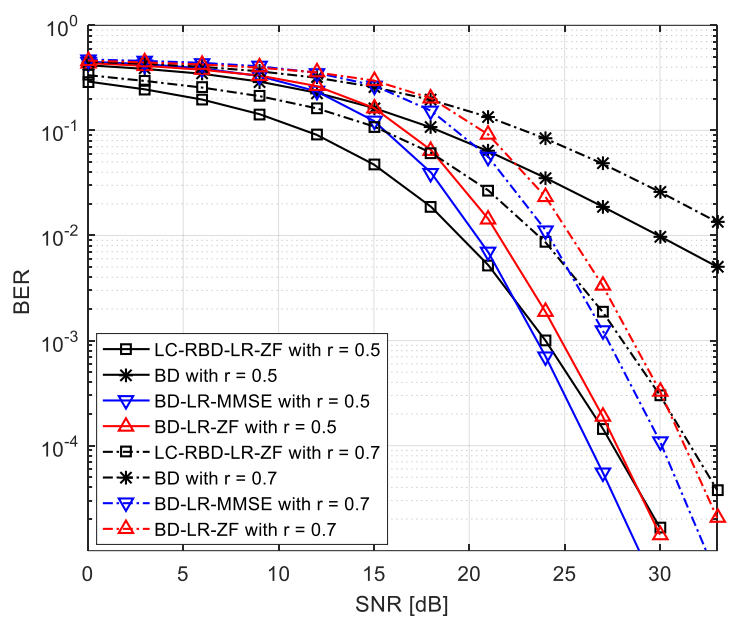

Fig. 6. The system performance with $N_{T}=8, N_{u}=2, K$ $=4$ in the case of correlated channel use the exponential correlation channel model, $r=0.5$ and $r=0.7$

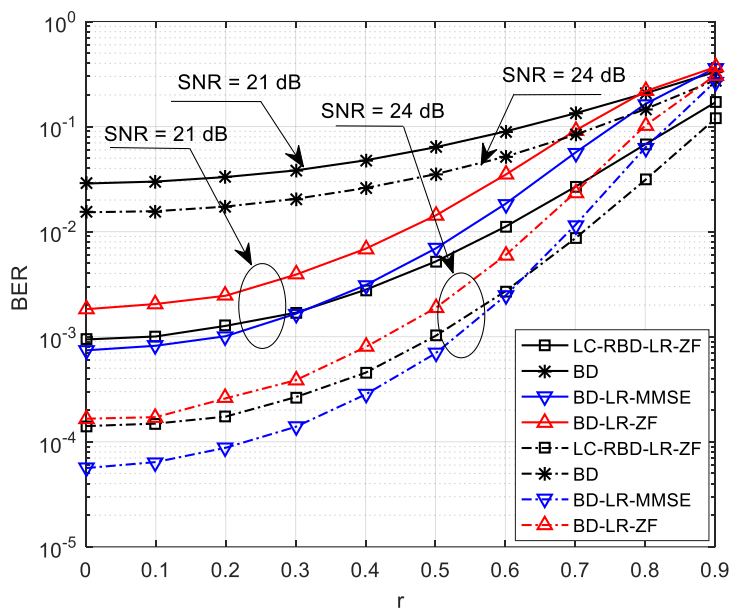

Fig. 7. The system performance according to $r$ at $\mathrm{SNR}=$

$21 \mathrm{~dB}$ and $24 \mathrm{~dB}$ with $N_{T}=N_{R}=8, K=4, N_{u}=2$

Fig. 7 illustrate the BER curves of all precoders as functions of $r$ at $\mathrm{SNR}=21 \mathrm{~dB}$ and $24 \mathrm{~dB}$. Other simulation parameters are the same as those used to generate Fig. 5, i.e., $N_{T}=N_{R}=8, K=4, N_{u}=2$, and 4QAM modulation. We can see that for the same parameters, BD precoder performs the worst. The remaining three precoders provide nearly the same BERs, particularly when $r$ becomes larger. Nevertheless, among the precoders, LC-RBD-LR-ZF precoder appears to be more robust as the correlation coefficient approaches unity. The simulation results in Fig. 7 also show that the correlation coefficient has an adverse effect on the system performance no matter which precoder is employed.

\section{Conclusions}

In this paper, we propose the BD-LR-ZF and BD-LRMMSE precoders by combining the conventional linear precoding techniques with low-complexity ELR-SLB lattice reduction technique to improve the BER performance of MU-MIMO systems under the exponential correlation channel model. It is shown that the BD-LR-ZF and BD-LR-MMSE precoders have remarkably lower complexity than their LC-RBD-LR-ZF and $\mathrm{BD}$ counterpart. In addition, the BER performances of the proposed algorithms are worse than the LC-RBDLR-ZF algorithm in the low SNR region, but better than the LC-RBD-LR-ZF algorithm in the high SNR region. $\mathrm{BD}$ precoder is shown to perform the worst among all the precoders. As a consequence, the proposed BD-LR-ZF and BD-LR-MMSE precoders can be potential digital beamforming techniques for practical MU-MIMO systems.

\section{References}

[1] H. Q. Ngo, Massive MIMO: Fundamentals and system designs. Linkoping University Electronic Press, 2015, vol. 1642

[2] T. L. Marzetta, "Noncooperative cellular wireless with unlimited numbers of base station antennas," IEEE Transactions on Wireless Communications, vol. 9, no. 11, pp. 3590-3600, November 2010.

[3] V. P. Selvan, M. S. Iqbal, and H. S. Al-Raweshidy, "Performance analysis of linear precoding schemes for very large multi-user mimo downlink system," Fourth edition of the International Conference on the Innovative Computing Technology (INTECH 2014), pp. 219-224, Aug 2014.

[4] Y. S. Cho, J. Kim, W. Y. Yang, and C. G. Kang, MIMOOFDM wireless communications with MATLAB. John Wiley \& Sons, 2010.

[5] Costa, "Writing on dirty paper," IEEE Transactions on Signal Processing, vol. 29, no. 3, 1983.

[6] O. Bai, H. Gao, T. Lv, and C. Yuen, "Low-complexity user scheduling in the downlink massive mu-mimo system with linear precoding," in 2014 IEEE/CIC International Conference on Communications in China (ICCC), Oct 2014, pp. 380-384.

[7] D. H. N. Nguyen, H. Nguyen-Le, and T. Le-Ngoc, "Blockdiagonalization precoding in a multiuser multicell 
mimo system: Competition and coordination," IEEE Transactions on Wireless Communications, vol. 13, no. 2, pp. 968-981, February 2014.

[8] H. An, M. Mohaisen, and K. Chang, "Lattice reduction aided precoding for multiuser mimo using seysen's algorithm," 2009 IEEE 20th International Symposium on Personal, Indoor and Mobile Radio Communications, pp. 2479-2483, Sept 2009.

[9] M. Simarro, F. Domene, F. J. MartAnez-Zald Avar, and A. Gonzalez, "Block diagonalization aided precoding algorithm for large mu-mimo systems," in 2017 13th International Wireless Communications and Mobile Computing Conference (IWCMC), June 2017, pp. 576581.

[10] W. Li and M. Latva-aho, "An efficient channel block diagonalization method for generalized zero forcing assisted mimo broadcasting systems," IEEE Transactions on Wireless Communications, vol. 10, no. 3, pp. 739-744, March 2011.

[11] K. Zu and R. C. d. Lamare, "Low-complexity lattice reduction-aided regularized block diagonalization for mumimo systems," IEEE Communications Letters, vol. 16, no. 6, pp. 925-928, June 2012.

[12] V. K. Dinh, M. T. Le, V. D. Ngo, X. N. Tran, and C. H. $\mathrm{Ta}$, "Transmit antenna selection aided linear group precoding for massive mimo systems," EAI Endorsed Transactions on Industrial Networks and Intelligent Systems, vol. 6, no. 21, 102019.

[13] V. K. Dinh, M. T. Le, V. D. Ngo, and C. H. Ta, "Pca-aided linear precoding in massive mimo systems with imperfect csi," Wireless Communications and Mobile Computing, vol. 2020, February 2020.

[14] R. N. A. Paulraj and D. Gore, Introduction to space-time wireless communications, New York: Cambridge University Press, 2003.

[15] S. L. Loyka, "Channel capacity of mimo architecture using the exponential correlation matrix," IEEE Communications Letters, vol. 5, no. 9, pp. 369-371, Sep. 2001.

[16] Q. Zhou and X. Ma, "Element-based lattice reduction algorithms for large mimo detection," IEEE Journal on Selected Areas in Communications, vol. 31, no. 2, pp. 274286, February 2013.

[17] M. Taherzadeh, A. Mobasher, and A. K. Khandani, "Lll reduction achieves the receive diversity in mimo decoding," IEEE Transactions on Information Theory, vol. 53, no. 12, pp. 4801-4805, Dec 2007.

[18] X. Ma and W. Zhang, "Performance analysis for mimo systems with lattice-reduction aided linear equalization," IEEE Transactions on Communications, vol. 56, no. 2, pp. 309-318, February 2008

[19] M. K. Simon, Probability Distributions Involving Gaussian Random Variables, Kluwer Academic Publishers, 2002.

[20] G. H. Golub and C. F. Van Loan, Matrix computations, Johns Hopkins Univ Press, 1996. 